\title{
Use of a thermal imager for snow pit temperatures
}

\author{
C. Shea $^{1}$, B. Jamieson ${ }^{1,2}$, and K. W. Birkeland ${ }^{3}$ \\ ${ }^{1}$ Department of Geoscience, University of Calgary, Calgary, Canada \\ ${ }^{2}$ Department of Civil Engineering, University of Calgary, Calgary, Canada \\ ${ }^{3}$ USDA Forest Service National Avalanche Center, Bozeman, Montana, USA \\ Correspondence to: C. Shea (cashea@ucalgary.ca)
}

Received: 6 September 2011 - Published in The Cryosphere Discuss.: 27 September 2011

Revised: 1 March 2012 - Accepted: 1 March 2012 - Published: 15 March 2012

\begin{abstract}
Weak snow of interest to avalanche forecasting often forms and changes as thin layers. Thermometers, the current field technology for measuring the temperature gradients across such layers - and for thus estimating the expected vapour flux and future type of crystal metamorphism - are difficult to use at distances shorter than $1 \mathrm{~cm}$. In contrast, a thermal imager can provide thousands of simultaneous temperature measurements across small distances with better accuracy. However, a thermal imager only senses the exposed surface, complicating its methods for access and accuracy of buried temperatures. This paper presents methods for exposing buried layers on pit walls and using a thermal imager to measure temperatures on these walls, correct for lens effects with snow, adjust temperature gradients, adjust time exposed, and calculate temperature gradients over millimetre distances. We find lens error on temperature gradients to be on the order of $0.03^{\circ} \mathrm{C}$ between image centre and corners. We find temperature gradient change over time to usually decrease - as expected with atmospheric equalization as a strong effect. Case studies including thermal images and visual macro photographs of crystals, collected during the 2010-2011 winter, demonstrate large temperature differences over millimetre-scale distances that are consistent with observed kinetic metamorphism. Further study is needed to use absolute temperatures independently of supporting gradient data.
\end{abstract}

\section{Introduction}

After depositing on the ground, the seasonal snowcover continues to change due to differences in vapour pressure within the ice lattice. With regard to avalanche formation, this metamorphism is of interest because it can indicate strengthening or weakening of the grain structure. A temperature difference between the surface of the snowpack and the ground generates a temperature gradient across the entire snowpack in a slope-normal direction. It is primarily this gradient, along with the snow structure, that drives differences in vapour pressure resulting in crystal metamorphism.

Practically, dry snow metamorphism is grouped into two categories: equilibrium and kinetic (Armstrong and Brun, 2008). The transition from equilibrium to kinetic metamorphism is generally accepted by field practitioners to occur at $1^{\circ} \mathrm{C}$ of temperature difference over $10 \mathrm{~cm}$ of distance (McClung and Schaerer, 2006). In the snow morphology model SNOWPACK, a more conservative value of $5^{\circ} \mathrm{C}$ per $1 \mathrm{~m}$ is empirically defined to be the transition between equilibrium and kinetic growth (Lehning et al., 2002), and this transition value appears in other work as well (Armstrong and Brun, 2008). Equilibrium metamorphism results in the rounding of snow crystals and strengthening of bonds. Kinetic metamorphism results in faceting, coarsening with average crystal size increase, and - potentially - weakening of structure.

However, Colbeck (1991) accurately, if indirectly, pointed out that in reality there is no such thing as true equilibrium, isothermal metamorphism. In the snowpack, any crystal metamorphism will result in vapour transport, latent heat exchange, and thus - at least microscopically - nonequilibrium temperature differences. To resolve this, the kinetic-equilibrium transition is deduced from macroscopic thermometer measurements, and any microscopic change in temperatures due to latent heat is often assumed to be absorbed and dissipated by the highly conductive ice lattice.

Recently, however, small snow samples in a uCT scanner show only $80 \%$ of the heat flux occurs through the ice lattice (Kaempfer et al., 2005). The same work also proposed that temperature gradients within these small samples varied 
widely from the globally applied gradient, and were not entirely absorbed and equalized by the lattice. Further, natural weak layers within the snowpack are often only a few millimetres thick (Heierli et al., 2008), suggesting that temperature gradients across small distances may be of interest. And temperature gradients - measured across distances of more than $1 \mathrm{~cm}-$ below the kinetic threshold have nevertheless yielded faceting and a coarse granular structure in the field (Jamieson, 2006; Smith et al., 2008).

Unfortunately, the hand held point thermometers currently used in the field are often inadequate for the task of discerning temperature differences across distances of a millimetre or measuring more than a handful of points. Modern thermal imagers, however, can provide sensitivity between pixels of better than $0.05^{\circ} \mathrm{C}$. Furthermore, these imagers can measure thousands of points in a two-dimensional array across a pit wall simultaneously and thus can limit the error introduced by individual measurements being spaced across time. As a thermal imager only senses the exposed surface, however, it is necessary to examine sources of error in snow pit measurements before using the imagers operationally.

This paper presents a discussion of error sources, their mitigation, and their correction when using a thermal imager specifically for obtaining temperature and gradient measurements on exposed pit walls. Afterwards, case studies introduce possible interpretations of such measurements.

\section{Previous work}

Temperature differences at these small distances - less than $2 \mathrm{~mm}$ - have not been previously measured or analyzed in a natural snowpack. In an artificially metamorphosed snowpack visualized with $\mu$ CT imaging, Kaempfer et al. (2005) modeled temperature differences across a $2.25 \times 2.7 \mathrm{~mm}$ slice of ice lattice subjected to a temperature gradient of $40^{\circ} \mathrm{C} \mathrm{m}^{-1}$ (when scaled to ${ }^{\circ} \mathrm{C}$ per $\mathrm{m}$ ). The model used to produce the resulting temperature field within the ice lattice shows gradients across sub-mm distances up to $510^{\circ} \mathrm{C} \mathrm{m}^{-1}$, even when ignoring the effects of phase change and thus latent heat. The authors note:

\begin{abstract}
"Analyzing the computed temperature distribution within the ice matrix, we note that high temperature differences of up to $20 \%$ of the overall $\Delta T$ occurred on a very short distance between one ice grain and another across a pore... This is due to the very tortuous structure of the ice matrix and leads to high temperature and vapor concentration gradients in the pores, which strongly influence metamorphism" (Kaempfer et al., 2005).
\end{abstract}

Miller (2002) proposed a similar crystal growth and thermal structure complexity, calling it a thermal short, like an electrical short circuit. Flin and Brzoska (2008) also computed a temperature field over a $3 \mathrm{~mm} \mu \mathrm{CT}$ imaged snow sample subject to a $16^{\circ} \mathrm{C} \mathrm{m}^{-1}$ global temperature gradient. Their work did not comment on the existence of significantly greater temperature differences at small distances; however, the goal of that work was not to describe heat flow, but rather to describe the equilibrium-kinetic transition using cellular automata-type growth laws.

Greene (2007) measured temperature differences in an artificial snowpack over time using thermocouples spaced around $10 \mathrm{~mm}$ apart, and with an assessed accuracy of around $0.3^{\circ} \mathrm{C}$. The resulting temperature profiles, even around the buried crust, were relatively smooth, and the author states: "all of the profiles show an element of linearity, but none are completely linear."

The findings of Kaempfer et al. (2005) seem very different than those of Flin and Brzoska (2008) and Greene (2007). On one hand, it seems easy to disregard the findings of Kaempfer et al. (2005) as an artifact of the model used; however, there are also practical observations of coarseness, faceting, and crust disaggregation occurring despite temperature gradients below the accepted $1{ }^{\circ} \mathrm{C}$ per $10 \mathrm{~cm}$ (Jamieson, 2006; Smith et al., 2008). The proposed reason for these observations is that stronger gradients exist at shorter distances, hidden within the larger measurable gradient as Kaempfer et al. (2005) observed from their model.

Small-scale temperature gradients also play roles in the design and execution of recent snow metamorphism models. Temperature gradients can be applied across a sample, globally, and the resulting temperature field and flux modelled, as in Kaempfer et al. (2005) and Flin and Brzoska (2008). Or, temperature gradients either drive flux and atomistic rules (Flin and Brzoska, 2008) or physical vapour pressure differences (Kaempfer and Plapp, 2009) which then result in predicted grain growth or sublimation. Kaempfer and Plapp (2009) achieve integration of latent heat exchange. But, to make such a model computationally tractable, assumptions about the computation step time need to be made based on the expected speed of vapour flow, which is in turn a function of microscopic temperature gradients. Obtaining accurate temperature gradient measurements across real - rather than modelled - pore spaces would be desirable to confirm and enhance these models.

Using thermal imagers for snow study is relatively new. Shea and Jamieson (2011) provide the first general overview, various applications, error sources, and correction specific to snow research. The use of a thermal imager to obtain temperatures across weak layers in a snow pit - the traditional use of a field temperature profile - has not yet been examined.

\section{Terminology}

A temperature gradient has three qualities: magnitude, distance, and direction. Some traditional terminology used in snow observation (McClung and Schaerer, 2006) refers to measurements as gradients when they do not occur on a 
scalar field. To reconcile practical and mathematical gradients, here we clarify terms used in this paper.

\subsection{Temperature gradient}

We retain the traditional use of temperature gradient to mean the difference in temperature between two points divided by the distance between them, as an absolute value. The real physical distance between the sample points - rather than simply a scaled ratio - remains important.

\subsection{Point gradient}

We use the term point gradient to mean the largest magnitude of difference, in any direction, from a single pixel within a thermal image to any of the pixel's eight nearest neighbors. Loosely, this corresponds to the image being a scalar temperature measurement field $f$, with a given pixel at $\left(x_{i}, y_{j}\right)$, giving the point gradient at that pixel to be $\left\|\nabla f\left(x_{i}, y_{j}\right)\right\|$, and reported along with the physical distance between the pixels.

\subsection{Row gradient}

As layers run slope-parallel within a natural snowpack, it is desirable to know the average gradient along a snow layer. Hence, when pixel rows in a thermal image also run slopeparallel, a row gradient is the average of the point gradients along a single row $y$ of pixels in a thermal image with $n$ columns:

$\frac{\sum_{i=1}^{n}\left\|\nabla f\left(x_{i}, y\right)\right\|}{n}$

This is of particular interest, for example, when the row aligns with the top or bottom interface of a snow layer. The row gradient would then provide an estimate of the average strength of flux at layer boundaries.

\subsection{Thin versus wide}

We use these descriptors to compare the distance between pixels in a point gradient or row gradient, or measurement points in a thermal gradient. For example, pixels with $1 \mathrm{~mm}$ between them measure a thinner temperature difference than thermometers spaced $5 \mathrm{~cm}$ apart. The distinction is necessary because, as demonstrated in Kaempfer et al. (2005) and the results in this paper, gradients are dependent on the distance scale.

\subsection{Strong versus weak}

These are relative descriptors for the absolute magnitude of temperature differences across a common distance. For example, a gradient of $1^{\circ} \mathrm{C}$ difference over $10 \mathrm{~cm}$ can be described as weaker than a gradient of $5^{\circ} \mathrm{C}$ difference over $10 \mathrm{~cm}$.

\section{Methods}

The method of using a thermal imager to obtain snow pit temperatures is itself a result. We developed these methods via field use over two seasons, and we offer them as a basis for further refinement. The current methods are:

1. Properly select and prepare a field study site (Sect. 5).

2. Clear the pit wall to a smooth vertical surface, and, as soon as possible, obtain multiple overlapping thermal images of the pit wall (Sect. 6).

3. Obtain a snow pit layer profile (Canadian Avalanche Association, 2007; Greene et al., 2010) with crystal morphology as a basis to assess future crystal changes. If needed, take an independent set of thermal images with landmarks (Sect. 6).

4. Mitigate or correct error in the voltages, temperatures, and gradients, both while in the field and through later analysis (Sect. 7).

5. Analyze and interpret the results (Sect. 8).

The following sections outline how we achieved these steps during the 2010-2011 season.

\section{Field study site}

The study site was a flat and open area located in the Canadian Rocky Mountains $\left(1650 \mathrm{~m}, 51.045^{\circ} \mathrm{N},-115.417^{\circ} \mathrm{W}\right)$. Between 28 January and 30 March 2011, 17 visits were made to obtain thermal infrared imagery of fresh pit walls. At 14 of those visits, visual macro crystal photographs were taken of snow crystals in every major layer and interface, both disaggregated and, when practical, in-situ.

Many attributes of this site allowed exploratory use of a thermal camera in obtaining thermal data in study pits (Fig. 1):

- The site was flat and without trees or bushes.

- The size of the area allowed for multiple pits in a single day to confirm continuity of the layer structure and twodimensional thermal pattern across the site.

- The shallow snowpack enabled every pit wall to be dug back farther than the snow depth to prevent edge effects from the previously exposed pit wall.

- The access allowed pit walls to face into the shade, mitigating heat input after exposure.

The shaded observation wall is desirable in any snow study pit. The other three attributes, however, were helpful in developing methods only, and so such a strict site selection would not necessarily be needed in the future. 


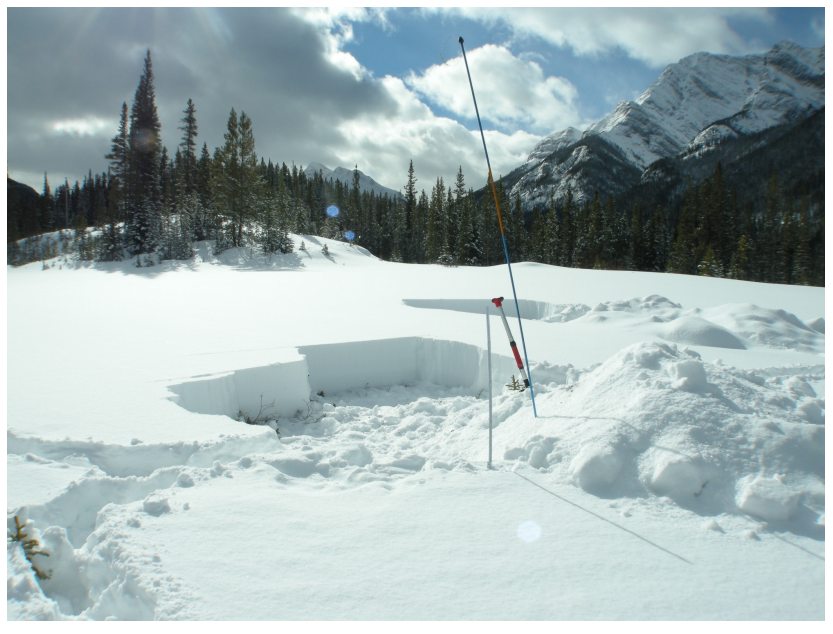

Fig. 1. The study site on 3 March 2011. Two pits were actively used, often on the same day, to confirm similarity of the layer structure across the site. Both pit walls stay in the shade due to careful orientation. The site provided enough space to dig back farther than the depth of the snowpack all winter. A shovel and $3 \mathrm{~m}$ long probe provide scale.

\section{Preparation}

With a suitable site, obtaining pit wall temperatures with a thermal camera was faster than obtaining them with a point thermometer. This needed to be so, to reduce error as described in Sect. 7. Field equipment included: a shovel with a flat blade edge, a thermometer for air temperature, and a thermal camera measuring infrared radiation between 7.5 and $13 \mu \mathrm{m}$. For improved atmospheric error correction, we sometimes used a humidity sensor, and a square of crumpled aluminum foil as described in Shea and Jamieson (2011) to obtain reflected apparent temperature. Alternatively, humidity was obtained from published local weather data, and cloud cover was recorded for later estimation of reflected apparent temperature. The camera used for all images in this paper was a FLIR B300 with a $320 \times 240$ pixel resolution.

We found that using a thermal camera to obtain temperature gradients on the surface of a pit wall is a skill set. One operator took all of the more than 2000 images using the thermal camera at this study site between 28 January and 30 March, and this operator had used the camera to obtain more than 1000 images using the same camera the previous season. The following skills for use of the camera in the field were identified:

1. The ability to consistently hold the camera aligned to the surface of the pit wall at $0^{\circ}$ of photographic angle. With practice using an inclinometer on the camera, this was achieved using a hand-held method. Elbows were steadied either on the ground, or on the operator's knees.

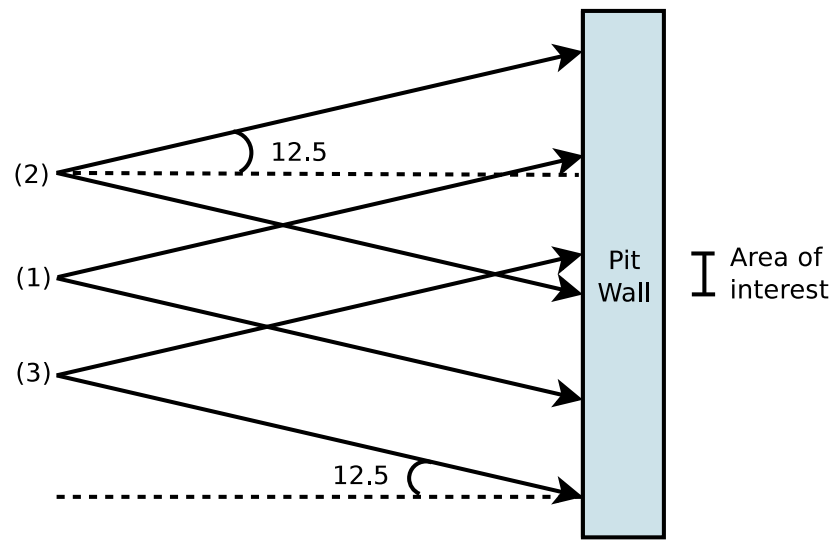

Fig. 2. Desired vertical overlap in thermal images, balancing the issues of area measured, time exposed, and lens error. The first image ideally would occur with the area of interest centered. To more completely assess surface changes over time at the area of interest, multiple images at position (1) can be taken.

2. The ability to quickly dig a planar pit wall. Shovel gouges and curved walls produce errors too large to easily correct. These gouges and irregularities were able to be clearly identified on the viewfinder after some experience, and if present, a new pit wall was dug.

3. The ability to take thermal images quickly. A streamlined field technique encompassing all steps from powering the camera on, to digging adequate area for the operator to work was developed. All data presented in this paper were obtained within ninety seconds of pit wall exposure.

\subsection{Field technique}

After the preparation above, we began digging and imaging pit walls. Intuitively, and for reasons described below in Sect. 7, the exposure time of the pit wall before being imaged should be minimized. We found it useful to expose a pit wall to within $20 \mathrm{~cm}$ of the surface to subsequently be imaged. This $20 \mathrm{~cm}$ buffer does not protect the snow for long - heat from an operator working close to a pit wall can penetrate more than $12 \mathrm{~cm}$ in $30 \mathrm{~min}$ (Shea and Jamieson, 2011) - but without it, timely measurement of an entire pit wall was often impossible. We would then remove the buffer iteratively to only expose an area that could be imaged in under one or two minutes. The camera would be powered on and allowed to equalize with the air to allow the camera to immediately be picked up and used as soon as each area was exposed.

Also for reasons described in Sect. 7, we took multiple images of the same area over time, overlapping by more than half the image height. Areas of particular interest, such as those at the top edge of a depth hoar layer, or small layers immediately above and below crusts, were included in the 
very first, fastest possible image. When possible, they were also centred in that first image, as shown in Fig. 2, to minimize lens effects as described in Sect. 7.2.

We developed various types of landmarks for later compositing multiple images into one larger image, or to mark the actual boundaries of a distinct layer. Often, the thermal structure of the snow itself provided sufficient landmarks. When these were absent, a ruler was placed at the edge of the images to provide a point of reference - often the paint ticks on the ruler could be read in the images due to their different thermal emissivity. With thin layers of different hardnesses, images for data were taken as soon as possible, and then at several points a finger, warmed pencil, or other tool was inserted into the softer layer and carefully run through the snow until it hit the harder interface, providing a landmark for the layer edge.

\section{$7 \quad$ Error corrections}

Some error, such as the operator heating described in Shea and Jamieson (2011), was best mitigated on-site rather than attempting corrections later. For example, in this paper, the time needed to take all of the images after pit wall exposure was minimized, and the operator would, when possible, lie on the snow with the camera between the operator's face and the snow pit wall to minimize the direct exposure of the pit wall to the operator.

Other errors, such as photographic angle, can be corrected for, but were more easily avoided by using a $0^{\circ}$ photographic angle for all images. The last category of error is that which could not be mitigated or avoided entirely, and this error includes atmospheric effects, lens effects with respect to snow, and time effects.

\subsection{Atmospheric effects}

Correction of atmospheric effects, discussed in Shea and Jamieson (2011), should be performed using the manufacturer's specifications because corrections occur on the raw voltages. Microbolometers are the sensors within thermal imagers, and they measure absorbed radiation within a given spectrum by changing resistance. The translation between a microbolometer sensor voltage and observed temperature, even with perfect emissivity, must be empirically developed. The translation is of course a function of the field variables (atmosphere temperature, reflected temperature, humidity, distance, and emissivity). But, it is also a function of the microbolometer temperature, as well as the physical nuances of the microbolometer itself. These physical engineering details - such as the attributes of the sensor hookups - would be inefficient or even impossible to derive physically, and so the relation as a whole is developed empirically. This calibration is performed at the thermal imager factory with a near-blackbody flat field and the microbolometer at known temperatures, and accounts for lens warp on a flat field.

As with any resistive temperature measuring device, within a microbolometer the connection to the individual pixel sensors also changes resistance based on the sensor temperature. In a microbolometer, the stable resistance over temperature must also be weighed against heat conductivity - to preserve as much heat as possible within the sensor itself - and manufacturing costs and limitations. These connections are often built from materials which give relatively stable measurements over a wide range of temperatures, varying their resistance and effect via heat conduction by around $2 \%$. Hence, when the ambient temperature of the microbolometer itself is not known, one can only have confidence in the absolute temperatures measured by a thermal imager to within $2 \%$. In practice, if the thermal imager is allowed to equalize with the ambient temperature, the temperature of the microbolometer can be known and accounted for as per the manufacturer's developed relation.

Also like any resistive temperature measuring device, when the pixel sensors are at a similar temperature to one another, measuring temperature difference between them is limited only by the voltage (or current) sensitivity of the circuits within the imager. For the FLIR B300, the sensitivity is enough to detect temperature-equivalent differences of $0.05^{\circ} \mathrm{C}$ or better. This is known as Noise Equivalent Temperature Difference (NETD). It applies between pixels within a single image, and between pixels in different images when the microbolometer is at a similar temperature. Hence, thermal imagers are subject to the approximately $2 \%$ absolute temperature accuracy limitation due to the physical characteristics of resistive devices, but more expensive thermal imagers can provide a better NETD by improving the voltage sensitivity of the measurement circuits. The high betweenpixel comparison accuracy allows gradients within and near layers of interest to be precisely examined, which is the primary application of interest to snow. Uses for these relative comparisons are shown in Sect. 8.

To correct for atmospheric effects, the voltages themselves are changed to account for field values and then the new, corrected voltages are translated via the factory-developed empirical relation to temperatures. For the FLIR B300 used in this paper, for example, the correction upon the voltages would be:

$V_{\text {obj }}=\frac{1}{\epsilon \tau} V_{\text {tot }}-\frac{1-\epsilon}{\epsilon} V_{\text {refl }}-\frac{1-\tau}{\epsilon \tau} V_{\text {atm }}$

Where $V_{\mathrm{obj}}$ is the measured voltage portion from the object (the snow), $V_{\text {tot }}$ is the total measured voltage at the microbolometer, $V_{\text {refl }}$ is the expected voltage portion seen atcamera from a blackbody with the reflected apparent temperature, and $V_{\text {atm }}$ is the expected voltage seen at-camera from a blackbody with the atmospheric temperature.

The field values are: $\epsilon$, which is the emissivity of the object averaged over the wavelengths of the sensor - a material 
property - and $\tau$, which is the transmittance of the intervening atmosphere. The camera operates in wavelengths of an atmospheric water vapor window, where $\tau \approx 1.0$, so the third term in Eq. (2) can be omitted.

By Kirchhoff's law, $1-\epsilon$ is the reflectivity of the snow surface. The reflected apparent temperature value within the portion is best measured by pointing the camera at a piece of crumpled aluminum foil, whose infrared reflectivity is very high, about 0.97 . Estimating by empirical methods (Sugita and Brutsaert, 1993) might be unreliable, because those methods were usually developed to estimate incoming atmospheric radiation over the entire infrared spectrum.

For the short distances used in this paper between thermal camera and pit wall $(50$ to $60 \mathrm{~cm}$ ), we use one measured value of reflected apparent temperature, one value of humidity, and drop the third term in Eq. (2). For emissivity of snow, the primary determinants are age, significant surface nonhomogeneity such as tall clumping, and wetness (Shea and Jamieson, 2011). Thus with non-fresh dry snow prepared to a relatively flat surface with a shovel, we used $\epsilon=0.98$ (Dozier and Warren, 1982) across the pit wall.

\subsection{Secondary lens effects}

Even with the imager pointing directly toward the pit wall a $0^{\circ}$ photographic angle - the lens itself is partially spherical with a $25^{\circ}$ field of view. Thus, effective photographic angles of up to $12.5^{\circ}$ occur in all images. Although for a flat field these lens effects are corrected for already, when imaging a complex snow surface, a lens changes both apparent temperatures and apparent gradients across pixels on a complex snow surface. The method of developing correction is emphasized, as each camera type lens may vary.

The reason for these secondary lens effects on gradients may be because a pit wall surface is not absolutely flat, and the roughness is slightly selective to crystal size - and therefore temperature differences. Smaller crystals shrink over time not only because of temperature gradient differences, but also because of curvature differences (Saito, 1996, p. 10). Thus, they sublimate faster in the snowpack and so cool more than their larger neighbors.

Depending on bond size, a sweep of a shovel blade may select some crystal sizes to remain at the very surface, and other crystal sizes to be be inset or tucked slightly behind the very surface of an exposed pit wall. Little protruding crystals may be chopped off with a shovel blade, exposing larger crystals, or larger crystals may be levered out, depending on the snowpack structure. When viewed straight on, as when an area of interest is framed in the centre of an image, the natural variation of crystals and their temperatures can be seen even when slightly inset and outset from the average surface plane. But away from the image centre, the slight lens angle may hide the inset crystal types and thus also artificially smooth the apparent gradient.
To develop this secondary correction, overlapping areas similar to Fig. 2 were identified in seven of the 17 days. The criteria for these seven days was very restrictive, as each overlapped area of interest needed to have an easily identifiable, natural landmark at all corners of the area. Furthermore, the overlapping image series needed to include either the first or second image taken after initial pit wall exposure. Each overlapping area contained approximately one-sixth to one-quarter of the pixels in a given individual image. The combination of the seven overlapping series covered all pixels on the lens.

After these seven overlapping areas were identified, voltages - and thus absolute temperatures - were corrected for atmospheric effects. Then, point gradients were calculated and normalized over each area. Finally, the distance of each area pixel was calculated from the centre of the original image. All distances were positive, so all four corners of the image were at a distance of 200 pixels. Finally, a linear model was built via ordinary least squares regression to relate the amount of change in point gradient per pixel distance from the image centre.

Based on the regression, the FLIR B300 shows a $1.5 \times 10^{-4}{ }^{\circ} \mathrm{C}$ decrease in normalized, atmospheric-corrected point gradient per pixel away from the centre of the image $\left(p<10^{-16}\right)$. This amounts to a $0.03^{\circ} \mathrm{C}$ decrease in point gradient at the extreme corners due to lens curvature and snow structure. As the camera has a between-pixel sensitivity noise of $0.05{ }^{\circ} \mathrm{C}$ (at worst), this is an acceptable - and correctable - amount of error.

Next, we assessed lens effect on absolute temperature. After the preparation steps outlined above, a linear model between pixel distance and normalized absolute temperature found $-2.57 \times 10^{-4}{ }^{\circ} \mathrm{C}$ of difference per pixel width away from the centre $\left(p<10^{-16}\right)$. This implies a $-0.051^{\circ} \mathrm{C}$ difference between centre and extreme corners, a similar value to the equipment sensitivity.

This can be compared to the photographic angle effects observed previously. The lens has a $12.5^{\circ}$ angle at the corners, or, using the relation above, $4 \times 10^{-3}{ }^{\circ} \mathrm{C}$ difference in temperature per degree angle of lens. This translates to $0.3^{\circ} \mathrm{C}$ difference projected over $75^{\circ}$, which is relatively similar to the $0.5^{\circ} \mathrm{C}$ difference calculated using photographic angle differences between $0^{\circ}$ and $75^{\circ}$ in Shea and Jamieson (2011).

\subsection{Exposure time effects}

At the very instant of exposure, the natural temperature structure is still present on the snow pit wall. In reality, some time always elapses before the first thermal image can be taken. Temperatures of the newly exposed pit wall may quickly be influenced by atmospheric effects. We wanted to assess whether the observed gradients were not artificially sharpening over time due to, for example, heat being conducted unevenly after exposure from behind the pit wall. 
Thirty-five pairs of overlap areas were identified. These pairs were from the same seven overlap areas used for lens correction earlier, to ensure that the areas were the most accurately corrected for effects other than time.

Of these thirty-five pairs, thirty-three had significantly different point gradient medians over time as measured by the Kolmogorov-Smirnov test. Of those thirty-three with significant differences, nine had increases in apparent gradient, and twenty-four had decreases. However, only one of those nine was an increase greater than the equipment sensitivity (an increase of $0.066^{\circ} \mathrm{C}, 0.016^{\circ} \mathrm{C}$ greater than the sensitivity). Seven of the twenty-four decreases were larger than the equipment sensitivity, even up to half of the initial gradient. On average, after being corrected for secondary lens effects as above, between-pixel temperature differences decreased $15 \%$ between an image taken as soon as possible after the pit wall being cleared and another within one minute later.

This change in median between-pixel temperature difference over time was independent of change in absolute temperature over time. Figure 3 shows lens-corrected and atmospheric-corrected temperatures which jump around quickly by as much as $0.4^{\circ} \mathrm{C}$ with no discernible pattern, but a steadily smoothing row gradient graph over time. This shows that although the snow surface may vary in temperature after exposure to the air, the relative temperatures of the snow generally follow a predictable pattern of homogenization.

The paired data imply that thin, crystal-scale point gradients usually decrease rather than increase in the ninety seconds after pit wall exposure. More than $90 \%$ of the time, we observed that the gradients on an exposed pit wall surface changed in a statistically significant manner within the first ninety seconds of exposure to the air. But only $23 \%$ of the time was that change larger than $0.05^{\circ} \mathrm{C}$, which is the sensitivity of the thermal imager. And less than three percent of the time did that change involve sharpening of the gradients rather than smoothing or no change over time.

This provides evidence that atmospheric equalization affected the surface temperature structure more strongly than, for example, heat from behind the snowpack coming forward unevenly. If the inverse were true, thermal gradients around layers with strong lateral conduction would increase after pit wall exposure to air, rather than decreasing. Indeed, as all images were obtained with some delay after pit wall exposure, these data also imply that even the strongest gradients in the first thermal images are weaker those present when first exposed, and therefore also diminished compared to the natural temperature structure.

To identify time-sharpening gradients in the future, the overlap from Fig. 2 would provide successive thermal images of one area. Increasing gradients can be easily identified either statistically or visually, and then either the most conservative gradient chosen or the data discarded.

Furthermore, the single instance of artificial gradient sharpening that was greater than equipment sensitivity oc- curred in a pair of images where the earlier image had the subject area at the very edge of the image (which smooths the apparent gradients, as discussed above), and the later image had the subject area at the very centre of the image. Although the gradients were corrected using the linear model as above, the model may not have provided adequate correction in this particular case. Centering the area of interest from the start and repeating the image with the area centred would provide an estimate of gradient sharpening least affected by the lens.

The expected and typical observed trend toward smoothing rather than sharpening of gradients over time makes physical sense despite the high bulk heat conductivity of snow. Lateral conduction capacity in mature, metamorphosed snow can reach such low levels that some models neglect lateral heat flux within the snowpack entirely (Greene, 2007).

\section{Case studies}

After field techniques and error correction were established, data from the field studies were examined. We present case studies of three selected examples from the data set below. These case studies offer possible interpretations for the data collected using a thermal imager, as well as suggesting that the point gradients observed represent not only real temperature differences but also temperature differences of importance to snow metamorphism.

\subsection{Scale dependence of temperature gradients}

On 29 January and 1 February 2011, we obtained thermal images and visual macro photographs of the facets and depth hoar interface below a buried crust. We compare the measured point gradients to the observed crystal growth.

\subsubsection{January 2011}

Multiple images were taken of the area of interest as described in Sect. 7.3, and the gradients were observed to decrease over time. The image with the least elapsed time after exposure was used. That thermal image was corrected for atmospheric effects as described in Sect. 7.1. Point gradients were calculated from these corrected temperatures, as described in Sect. 3. Both the temperatures and gradients were corrected for lens effects (Sect. 7.2) and are displayed in Fig. 4. A selection of row gradients are also shown in Fig. 4.

The large point gradients in Fig. 4, which exceed $2{ }^{\circ} \mathrm{C}$ difference over a $1.8 \mathrm{~mm}$ pixel width, are essentially hidden to the traditional field temperature measurement method. The average temperature along the top of the thermal image in Fig. 4 is $-7.41^{\circ} \mathrm{C}$, and the average temperature along the bottom is $-6.74{ }^{\circ} \mathrm{C}$. Therefore, on average, placing a point thermometer at the top, and a point thermometer at the bottom, would reveal a temperature difference of only $0.67^{\circ} \mathrm{C}$ 

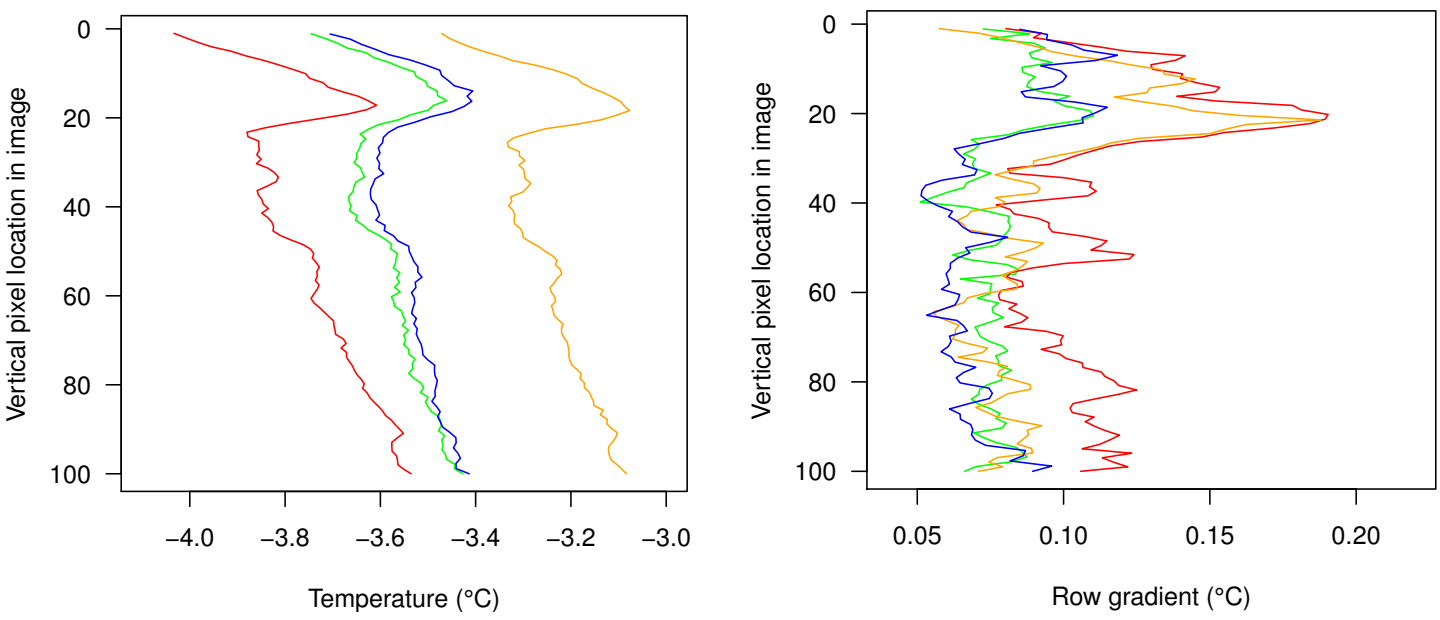

Fig. 3. (a) Temperature fluctuations over time, and (b) gradient fluctuations over time, from the same area. Both graphs have lines coloured in rainbow order of the time the photo was taken. Red first, then orange, then green, then blue. The time between the red line image and the blue line image is approximately one minute, whereas the red and orange images are approximately ten seconds apart at most.

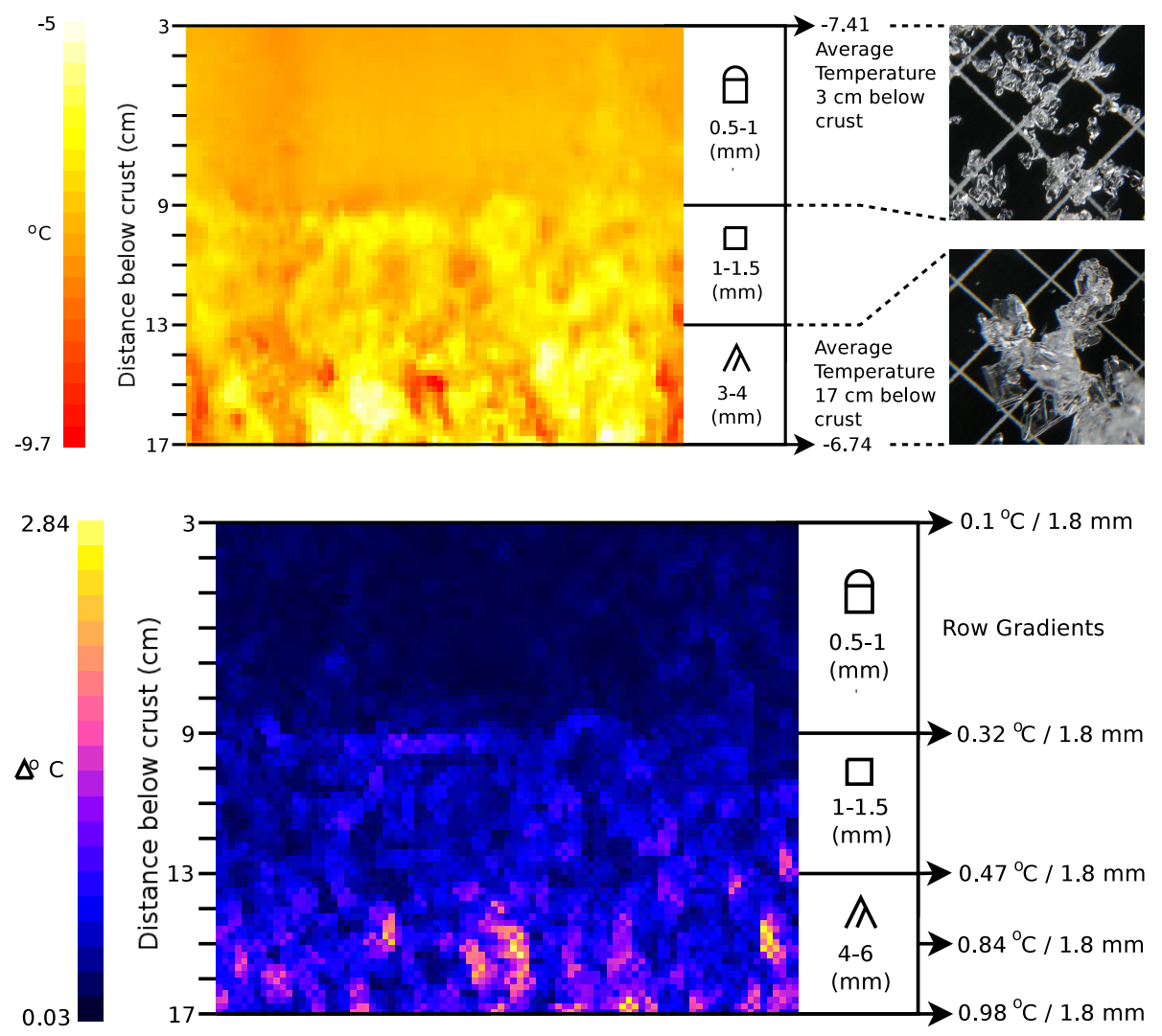

Fig. 4. Temperatures and point gradients at the leading edge of the depth hoar on 29 January 2011. The grids on the crystal screen have $3 \mathrm{~mm}$ spacing. Crystal types are from Fierz et al. (2009).

over $14 \mathrm{~cm}$. This is less than the accepted $10^{\circ} \mathrm{Cm}^{-1}$ assumed boundary for kinetic metamorphism. It is also less than the more conservative modeled boundary for kinetic metamorphism at $5^{\circ} \mathrm{C} \mathrm{m}^{-1}$ in Lehning et al. (2002). With the thermal imager, however, a row gradient of $0.98^{\circ} \mathrm{C}$ over
$1.8 \mathrm{~mm}$ can be measured at the lower edge, which, scaled to the metre, is $540^{\circ} \mathrm{C} \mathrm{m}^{-1}$. 


\subsubsection{February 2011}

These point and row gradients can be compared to the change in physical crystal size, as well as to their relative depth from the horizontally even crust. The crust provided the most accurate landmark as new snow had fallen and the ground was somewhat uneven. Crystal changes and their depth changes can be seen in Fig. 5. Despite the lack of large temperature difference over the $14 \mathrm{~cm}$ height of the image, it is clear that facet growth and therefore kinetic metamorphism occurred after the large point gradients were observed on 29 January, particularly in the depth hoar layer where the large point and row gradients were observed.

\subsubsection{Interpretation}

These thin point temperature gradients could be caused by:

- Tortuosity of the snow structure, as proposed by Kaempfer et al. (2005).

- Remnants of undissipated latent heat flux on the crystals from previous metamorphism (which, upon affecting the temperature, will affect future metamorphism).

These point gradients can be further interpreted as the cause of the observed growth by estimating how much ice mass may be moved by them, and whether that mass concurs with the observed changes. Using a gross estimation of 1:50 for crystal length per unit thickness (Libbrecht, 2005) of an ice crystal, we can estimate that the average large crystal in the depth hoar on 29 January is about $3 \times 3 \times 0.06 \mathrm{~mm}=0.54 \mathrm{~mm}^{3}$, and on 1 February it is $4 \times 4 \times 0.08 \mathrm{~mm}=1.28 \mathrm{~mm}^{3}$.

With an ice density of $9.2 \times 10^{-4} \mathrm{~g} \mathrm{~mm}^{-3}$, the change between the estimated crystal on 29 January to the estimated crystal on 1 February is $0.74 \mathrm{~mm}^{3}$, or $6.8 \times 10^{-4} \mathrm{~g}$. Use of Fick's Law (Stull, 1995, p. 133) conserves and moves mass over the vapour phase and describes the diffusion flux by vapour density difference as a function of temperature:

$F=-D \frac{\delta \rho}{\delta z}$

Here, $z$ is the physical distance between pixel centres, $D$ is the diffusion coefficient for water vapour $(2.22 \times$ $\left.10^{-5} \mathrm{~m}^{2} \mathrm{~s}^{-1}\right), F$ is the flux, and $\rho$ is the density of the water vapour in the air in $\mathrm{g} \mathrm{m}^{-3}$. Saturation vapour pressure $e_{\mathrm{s}}$ in $\mathrm{J} \mathrm{m}^{-3}$ at a given air temperature $T$ may be found by starting with the Clausius-Clapeyron approximation equation (Stull, 1995, p. 84):

$e_{\mathrm{S}}=611 \mathrm{~Pa} \cdot \exp \left\{\frac{L_{\mathrm{d}}}{R_{\mathrm{V}}}\left(\frac{1}{273 \mathrm{~K}}-\frac{1}{T}\right)\right\}$

Here, $L_{\mathrm{d}}$ is the latent heat of deposition of water $\left(2.83 \times 10^{6} \mathrm{~J} \mathrm{~kg}^{-1}\right)$ and $R_{\mathrm{v}}\left(461 \mathrm{~J} \mathrm{~K}^{-1} \mathrm{~kg}^{-1}\right)$ is the specific

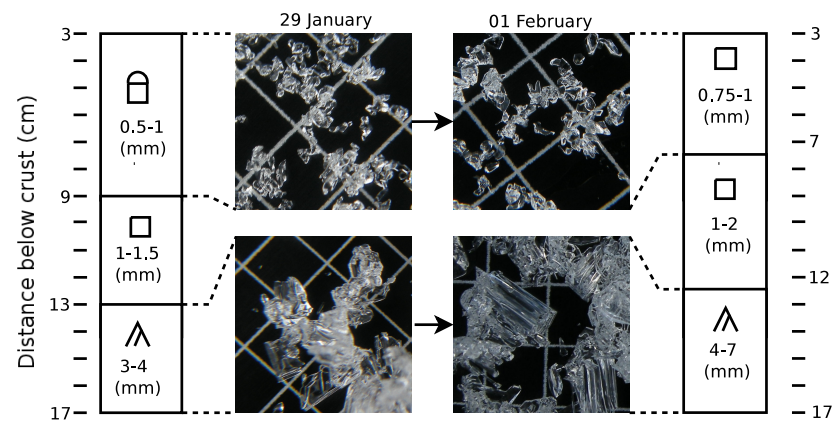

Fig. 5. Crystal changes subsequent to the gradients observed on 29 January 2011. The tops of the facet and depth hoar layers also grew closer to the crust, from 9 and $13 \mathrm{~cm}$ below the crust, respectively, to 7.5 and $12.5 \mathrm{~cm}$ below the crust. The grids on the crystal screen have $3 \mathrm{~mm}$ spacing. Crystal types are from Fierz et al. (2009).

water vapour gas constant. Then, the ideal gas law gives the density $\rho$ needed for Eq. (3):

$e_{\mathrm{S}}=\rho R_{\mathrm{v}} T$

Thus we can approximate the diffusive flux, $F$, by obtaining the difference in expected vapour density, $\delta \rho$, from one point gradient and the absolute temperature at that point. The flux is across the pore space, from ice, through air, to ice.

On 29 January, we selected typical values from the bottom row of the image having $-6.74{ }^{\circ} \mathrm{C}$ corrected temperature and $-0.98^{\circ} \mathrm{C}$ corrected row gradient over $1.8 \mathrm{~mm}$. This creates a flux vector between $-6.74{ }^{\circ} \mathrm{C}(266.42 \mathrm{~K})$ and $-7.72^{\circ} \mathrm{C}$ $(264.4 \mathrm{~K})$ over $1.8 \times 10^{-3} \mathrm{~m}$. From Eqs. (3) through (5) and converting to $\mathrm{g} \mathrm{m}^{-3}$ in Eq. (5), saturation vapour density over ice at $266.42 \mathrm{~K}$ is approximately $2.82 \mathrm{~g} \mathrm{~m}^{-3}$, and at $264.4 \mathrm{~K}$ it is $2.60 \mathrm{~g} \mathrm{~m}^{-3}$. This will create flux in the pore space of $F=2.69 \times 10^{-3} \mathrm{~g} \mathrm{~m}^{-2} \mathrm{~s}^{-1}$.

Over $70 \mathrm{~h},\left(2.5 \times 10^{5} \mathrm{~s}\right)$, through a two-dimensional area the size of the crystal $\left(3 \times 3 \mathrm{~mm}\right.$ wide, or $\left.9 \times 10^{-6} \mathrm{~m}^{2}\right)$, this flux will move $6.1 \times 10^{-3} \mathrm{~g}$ of water, about ten times that needed to grow the smaller crystal to the larger one. But this is just the water that moves, not that which attaches to the new ice surface. The proportion of transported water vapour which actually attaches is very roughly estimated to be between one tenth to one one thousandth of the transported vapour (Kaempfer and Plapp, 2009), which is consistent with the range of observed growth between 29 January and 1 February in the depth hoar layer. Further, only an initial temperature gradient value was used; hence, at best only a comparable range can be expected.

These observed growth rates also show that latent heat may not easily be ignored. The amount of latent heat that this deposition would add to the original crystal, which was at an average of $-7^{\circ} \mathrm{C}$ on 29 January (Fig. 4), is $2260 \mathrm{~J} \mathrm{~g}^{-1}$, or $1.54 \mathrm{~J}$ over the intervening $70 \mathrm{~h}$. Assuming $80 \%$ of this heat flux travels and is dispersed through the conduction of 

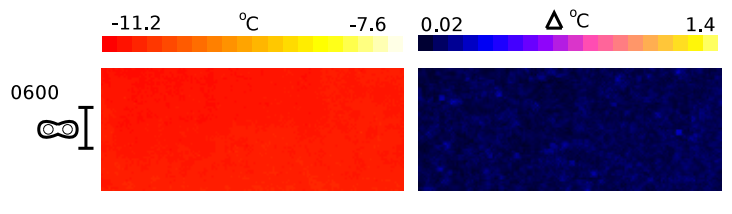

1.4
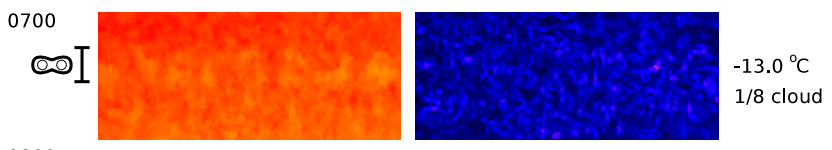

0800
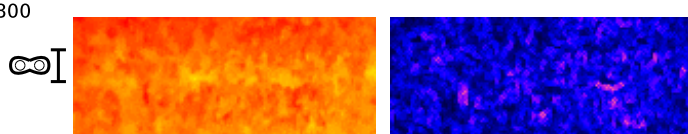

$-15.6{ }^{\circ} \mathrm{C}$ $0 / 8$ cloud
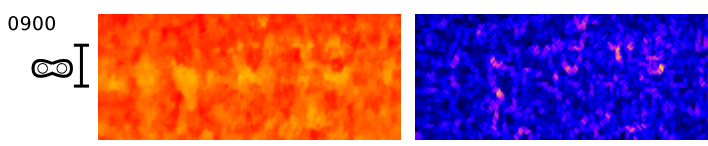

$-14.5^{\circ} \mathrm{C}$

$1 / 8$ cloud
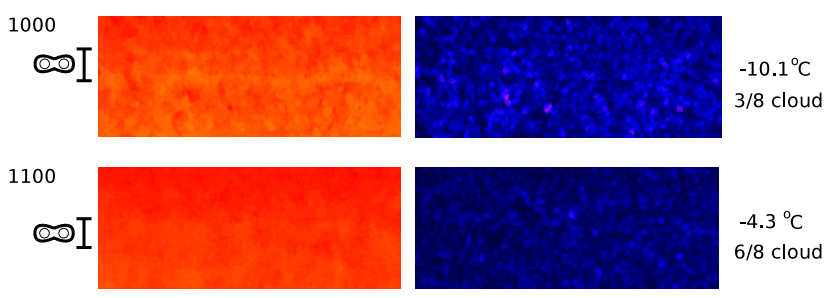

$-4.3^{\circ} \mathrm{C}$

$6 / 8$ cloud

Fig. 6. Temperatures (left images) and point gradients (right images) over five hours on the morning of 3 March 2011. Air temperatures and cloud cover are listed on the far right. The hour of measurement and crust location (vertical placement and $1 \mathrm{~cm}$ height) are noted on the far left.

the ice matrix (Kaempfer et al., 2005), this leaves $20 \%$, or $0.31 \mathrm{~J}$ which will stay, warm the crystal, and drive further water vapour transport upwards through the pore space.

This $0.31 \mathrm{~J}$ is a large increment of energy for a small crystal. Ice only takes $2.11 \mathrm{~J} \mathrm{~g}^{-1}$ to raise its temperature by $1^{\circ} \mathrm{C}$, or $1 \times 10^{-3} \mathrm{~J}$ per degree for the crystal mass. If the temperatures on crystals during metamorphism did not fluctuate to achieve steady state and satisfy the global heat flux gradient across the snowpack and at the same time minimize the gradients across individual pore spaces, the point gradients in Fig. 4 would not show a maximum of $2.84^{\circ} \mathrm{C}$ between pixels. Rather, the difference would be much greater due to the undissipated latent heat alone at these observed crystal growth rates.

\subsection{Temporally changing temperature gradients}

On 3 March 2011, we obtained thermal images every hour at the study site. Here, we focus on the images obtained during a period of sky cooling and clearing in the morning, during which relatively strong point temperature gradients appeared around a buried crust and then disappeared again within hours.

\subsubsection{March 2011}

Thermal images of the buried crust $(27.5 \mathrm{~cm}$ depth from surface, $1 \mathrm{~cm}$ layer vertical height) were taken every hour during the day of 3 March 2011. Each time, a fresh pit wall was dug farther back than the snow depth. Multiple images of each area showed sequential gradient smoothing, and the very first image in each series is used here for analysis. These images were corrected for atmospheric effects and then the portion of the image containing the crust was extracted. Point gradients were calculated within these sub-areas and both temperatures and gradients were corrected for lens effects. The results of this series between 06:00 and 11:00 LT, may be seen in Fig. 6 .

\subsubsection{Interpretation}

The reason for these transient, pore-space-scale temperature gradients could be latent heat release due to new ice deposition. This deposition may have resulted from increased upward heat - and therefore vapour - flux during external cooling of the snowcover surface from 06:00 to 09:00 LT.

The attribute most supporting the latent heat explanation is the warming of the crust during the cooling of the atmosphere. During this cooling, the point gradients throughout the whole area are increasing, implying active vapour transport. This also indicates that vapour is transferring heat in larger amounts than the conductive ice lattice can handle. As this episode is brief enough to not significantly affect the ice mass and thus tortuosity within the area, the point gradients can be dissipated - via conduction - when the vapour flow ebbs as the outside cloud cover returns.

This temporally short instance of gradients appearing on the micro scale due to increased flux on the macro scale implies that the formation of gradients - which in turn drive metamorphism - are not only a function of the current difference of temperature from ground to snow surface, but also how conductive the ice lattice has become during past metamorphism to handle the heat flux. Flux above and beyond what the ice lattice can conduct may push more flux through the pores, resulting in latent heat release via deposition, changing local temperature differences, and thus possibly affecting the type of crystal growth that results or even type of metamorphism.

Although some studies such as Smith et al. (2008) and Greene (2007) obtain many measurements over short time periods - yet did not find evidence of strong temperature differences across crusts despite coarsening of the granular structure around the crust - practitioners usually only record point temperatures in the field once per day and then only at 5 to $10 \mathrm{~cm}$ distances. It may be difficult to discover these times of locally strong point gradients, as well as - more importantly - discovering at what level of snowpack-wide heat flux their conduction capacities are approached, resulting in significant vapour transport through the pores. 


\subsection{Sub-surface heating}

On 25 March 2011, we obtained thermal images of solar heating below, but not at, the snow surface, also known colloquially as sub-surface heating, or the solid state greenhouse effect (Brandt and Warren, 1993). This resulted in near-surface faceting of the radiation recrystallization type (LaChapelle and Armstrong, 1977), shown by crystal macro photographs on 30 March 2011, and described below.

\subsubsection{March 2011}

The first thermal image obtained as soon as the pit wall was exposed was used for analysis. These temperatures were corrected for atmospheric effects, and then point gradients were calculated and both were corrected for secondary lens effects. The temperatures and gradients appear in Fig. 7.

The thermal image in Fig. 7 was taken much closer to the pit wall than previous images in this paper; each pixel represents $0.8 \mathrm{~mm}$. The row gradient above the warmed area is $0.20^{\circ} \mathrm{C}$, and the row gradient below is $0.122^{\circ} \mathrm{C}$. These translate to $250^{\circ} \mathrm{C} \mathrm{m}^{-1}$ and $150^{\circ} \mathrm{C} \mathrm{m}^{-1}$ respectively, when scaled to ${ }^{\circ} \mathrm{C} \mathrm{m}^{-1}$. The maximum point gradient, which is above the warmed area, is $0.47^{\circ} \mathrm{C}$, or an equivalent $580^{\circ} \mathrm{C} \mathrm{m}^{-1}$.

\subsubsection{March 2011}

Unfortunately, we could not return to the site to document change in crystal morphology until five days later. On 30 March, the interfaces both above and below the warmed layer had developed faceted crystals as shown in Fig. 7. Also on 30 March, the warmed area itself had formed grains of melt-freeze crust (MFcr) between 3 and $7 \mathrm{~mm}$ (Fierz et al., 2009).

The faceting observed on 30 March above and below this sub-surface warmed layer was expected from the strong point temperature gradients measured on 25 March. The size and maturity of the facets also corresponded to the previous relative strengths of the point and row gradients above and below. The facets above the warmed layer - corresponding to the stronger point and row gradients - were larger and sharper than those under the layer. These upper facets also displayed evidence of near-surface faceting protrusions which were not present on the facets below the warmed layer.

\subsubsection{Interpretation}

Many explanations already exist for the faceting above and below this warmer and harder layer. Colbeck (1991) proposed that sharp vapour gradients would appear above and below a denser layer and grow facets via kinetic metamorphism. Strong temperature gradients at the interface of a warm, near-freezing layer have been measured using thermocouples (Fukuzawa and Akitaya, 1993; Jamieson and Fierz, 2004) and the associated faceting modelled in bulk (Jamieson and Fierz, 2004). Near-surface temperature gradients on

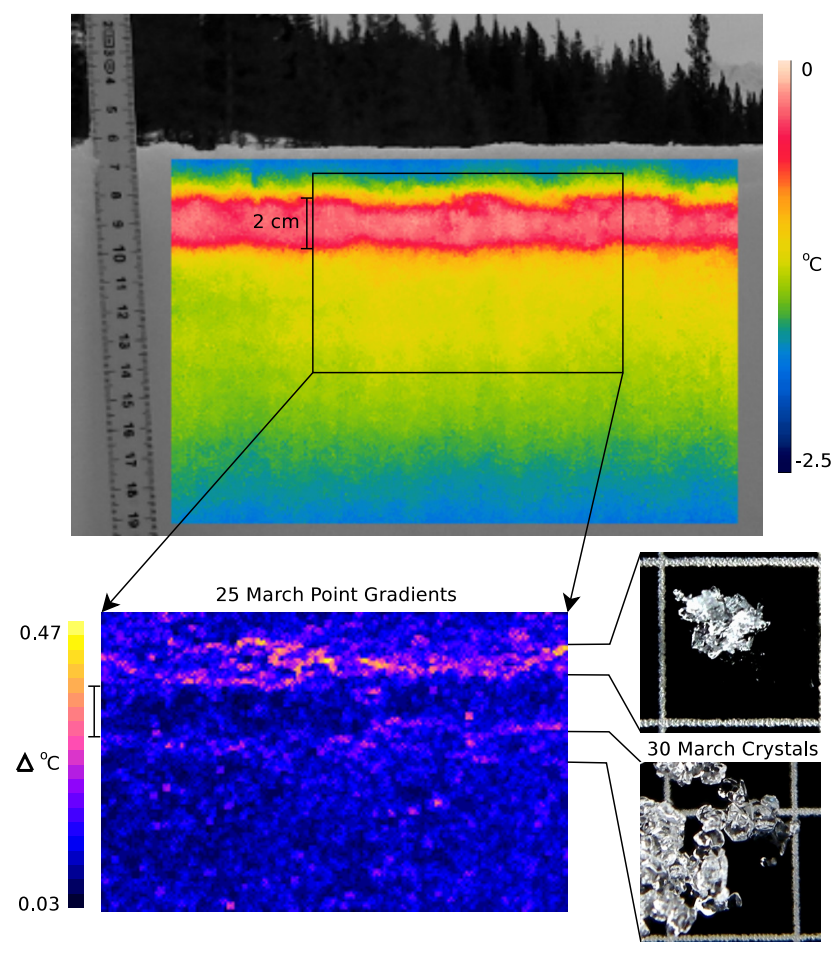

Fig. 7. Temperatures and point gradients during near-surface warming. The temperatures and point gradients are from $25 \mathrm{March}$; the crystal macro photographs are from 30 March on a $3 \mathrm{~mm}$ grid. In both upper and lower images, a span bar on the left denotes the same physical area.

the order of $250^{\circ} \mathrm{Cm}^{-1}$, even with the warmed layer below freezing, have also been measured along with associated faceting due to radiation recrystallization (Birkeland et al., 1998).

However, here the thermal imager provides a new perspective of two-dimensional continuity. As weak layers benefit from lateral homogeneity to propagate fractures (Heierli et al., 2008), in the future the continuity or discontinuity of these point gradients could provide additional information for the analysis of weak layer metamorphism and stability.

\section{Summary and conclusions}

Thermal images of a pit wall showed point temperature gradients in excess of $500^{\circ} \mathrm{C} \mathrm{m}^{-1}$ in a snowpack structure which would present only a $0.67^{\circ} \mathrm{C}$ difference between two thermometers spaced $14 \mathrm{~cm}$ apart in the same area. Such extreme, thin gradients hidden within a weaker, wider temperature difference fits well with the modelling of Kaempfer et al. (2005). Such gradients also provide an explanation for observed crystal changes not explained by temperature differences over 5 to $10 \mathrm{~cm}$ (Jamieson, 2006; Smith et al., 2008). Although there is large uncertainty in the calculation due, in particular, to the attachment rate, the initial point gradients 
were consistent with the vapour transport needed for the crystal growth observed over the three days afterwards.

During sky clearing and cooling, point gradients in excess of $1{ }^{\circ} \mathrm{C}$ between adjacent pixels $1-2 \mathrm{~mm}$ wide were found to develop within hours around a crust buried $27 \mathrm{~cm}$ below the surface. These gradients subsequently disappeared during an increase in cloud cover. These thin, transient gradients have not been previously documented, although they offer a possible explanation for the field observations of nearcrust faceting not explained by traditional temperature profiles measured at a point in time (Jamieson, 2006).

In the case of the near-surface warming presented, the row gradient values have been observed at similar magnitudes using thermocouples (Birkeland et al., 1998; Fukuzawa and Akitaya, 1993), but the magnitude of gradients between individual pixels have not been previously observed. Much remains to be done via future work in establishing what values of point gradients have what effect on eventual morphology. This, along with the first case study, are both instances when gradients measured by a thermal imager were indicative of subsequently observed metamorphism.

Building on the mitigations and concerns from Shea and Jamieson (2011), images needed to be corrected for atmospheric effects. A series of images of the same area were taken as soon as possible after each pit wall exposure to assess specific changes in gradients over time, and pit wall gradients typically reduced within the first ninety seconds. Future work needs to assess the usefulness of absolute temperatures independently of between-pixel gradients.

The thermal camera and the methods described in this paper provided data with a quantitative accuracy and spatial resolution currently not achievable by any other means. This type of data may, in the future, help link modelling of metamorphism and thin temperature gradients to the same occurrences in the natural snowcover.

Acknowledgements. For financial support, the authors thank the Natural Sciences and Engineering Research Council of Canada, HeliCat Canada, the Canadian Avalanche Association, Mike Wiegele Helicopter Skiing, Teck Mining Company, Canada West Ski Areas Association, the Association of Canadian Mountain Guides, Backcountry Lodges of British Columbia, and the Canadian Ski Guides Association.

We thank reviewers Jeff Dozier, Zoe Courville, and Panu Lahtinen for their comments which have not only improved this paper, but also - as part of the open review process - enhanced this paper above and beyond its content alone. We also thank editor Shawn Marshall for his expert handling of the diverse input and discussion during the review process.

Edited by: S. Marshall

\section{References}

Armstrong, R. L. and Brun, E.: Snow and Climate: Physical Processes, Surface Energy Exchange and Modeling, Cambridge University Press, Cambridge, United Kingdom, 2008.

Birkeland, K., Johnson, R., and Schmidt, D.: Near surface faceted crystals formed by diurnal recrystallization: A case study of weak layer formation in the mountain snowpack and its contribution to snow avalanches, Arctic Alpine Res., 30, 200-204, 1998.

Brandt, R. E. and Warren, S. G.: Solar-heating rates and temperature profiles in Antarctic snow and ice, J. Glaciol., 39, 99-110, 1993.

Canadian Avalanche Association: Observation Guidelines and Recording Standards for Weather, Snowpack and Avalanches, Canadian Avalanche Association, Revelstoke, British Columbia, Canada, 2007.

Colbeck, S.: The layered character of snow covers, Rev. Geophys., 29, 81-96, 1991.

Dozier, J. and Warren, S. G.: Effect of Viewing Angle on the Infrared Brightness Temperature of Snow, Water Resour. Res., 18, 1424-1434, 1982.

Fierz, C., Armstrong, R., Durand, Y., Etchevers, P., Greene, E., McClung, D., Nishimura, K., Satyawali, P., and Sokratov, S.: The International Classification for Seasonal Snow on the Ground, IHP-VII Technical Documents in Hydrology N. 83, IACS Contribution N. 1, UNESCO-IHP, Paris, 2009.

Flin, F. and Brzoska, J.: The temperature-gradient metamorphism of snow: vapour diffusion model and application to tomographic images, Ann. Glaciol., 49, 17-21, 2008.

Fukuzawa, T. and Akitaya, E.: Depth-hoar crystal growth in the surface layer under high temperature gradient, Ann. Glaciol., 18, 39-45, 1993.

Greene, E.: The Thermophysical and Microstructural Effects of an Artificial Ice Layer in Natural Snow Under Kinetic Growth Metamorphism, Ph.D. Thesis, Department of Geoscience, Colorado State University, Fort Collins, Colorado, USA, 2007.

Greene, E., Atkins, D., Birkeland, K., Elder, K., Landry, C., Lazar, B., McCammon, I., Moore, M., Sharaf, D., Sternenz, C., Tremper, B., and Williams, K.: Snow, Weather, and Avalanches: Observational Guidlines for Avalanche Programs in the United States, American Avalanche Association, Pagosa Springs, Colorado, USA, Second Printing Fall 2010, 2010.

Heierli, J., Gumbsch, P., and Zaiser, M.: Anticrack nucleation as triggering mechanism for snow slab avalanches, Science, 321, 240-243, 2008.

Jamieson, B.: Formation of refrozen snowpack layers and their role in slab avalanche release, Rev. Geophys., 44, RG2001, doi:10.1029/2005RG000176, 2006.

Jamieson, B. and Fierz, C.: Heat flow from wet to dry snowpack layers and associated faceting, Ann. Glaciol., 38, 187-194, 2004.

Kaempfer, T. and Plapp, M.: Phase-field modeling of dry snow metamorphism, Phys. Rev., 79, 031502, 1-17, 2009.

Kaempfer, T., Schneebeli, M., and Sokratov, S.: A microstructural approach to model heat transfer in snow, Geophys. Res. Lett., 32, L21503, doi:10.1029/2005GL023873, 2005.

LaChapelle, E. and Armstrong, R.: Temperature patterns in an alpine snow cover and their influence on snow metamorphism, U.S. Army Research Office, Institute of Arctic and Alpine Research Technical Report, University of Colorado, 1977.

Lehning, M., Bartelt, P., Brown, B., and Fierz, C.: A physical 
SNOWPACK model for the Swiss avalanche warning Part III: meteorological forcing, thin layer formation and evaluation, Cold Reg. Sci. Technol., 35, 169-184, 2002.

Libbrecht, K.: The physics of snow crystals, Rep. Prog. Phys., 68, 855-895, 2005.

McClung, D. and Schaerer, P.: The Avalanche Handbook, Third Edition, The Mountaineers Books, Seattle, WA, USA, 2006.

Miller, D.: An Integrated Microstructural Study of Dry Snow Metamorphism under Generalized Thermal Conditions, Ph.D. Thesis, Department of Engineering, Montana State University, Bozeman, Montana, USA, 2002.

Saito, Y.: Statistical Physics of Crystal Growth, World Scientific, 1996.
Shea, C. and Jamieson, B.: Some fundamentals of handheld snow surface thermography, The Cryosphere, 5, 55-66, doi:10.5194/tc-5-55-2011, 2011.

Smith, M., Jamieson, B., and Fierz, C.: Observation and modeling of buried melt-freeze crusts, in: Proceedings of the 2008 International Snow Science Workshop in Whistler, BC, Canada, 170178, 2008.

Stull, R. B.: Meteorology Today for Scientists and Engineers, West Publishing Company, St. Paul, Minnesota, USA, 1995.

Sugita, M. and Brutsaert, W.: Cloud effect in the estimation of instantaneous downward longwave radiation, Water Resour. Res., 29, 599-605, 1993. 